\title{
Immune Checkpoint $\mathrm{B} 7 \mathrm{x}$ (B7-H4/B7S1/VTCN1) is Over Expressed in Spontaneous Canine Bladder Cancer: The First Report and its Implications in a Preclinical Model
}

\author{
Damini Chand ${ }^{\mathrm{a}, \mathrm{b}}$, Deepika Dhawan ${ }^{\mathrm{c}}$, Alexander Sankin ${ }^{\mathrm{b}}$, Xiaoxin Ren ${ }^{\mathrm{a}}$, Juan Lin ${ }^{\mathrm{d}}$, \\ Mark Schoenberg ${ }^{\mathrm{b}}$, Deborah W. Knapp ${ }^{\mathrm{c}, \mathrm{e}}$ and Xingxing Zang ${ }^{\mathrm{a}, \mathrm{b}, *}$ \\ ${ }^{a}$ Department of Microbiology and Immunology, Albert Einstein College of Medicine, Bronx, NY, USA \\ ${ }^{\mathrm{b}}$ Department of Urology, Montefiore Medical Center and Albert Einstein College of Medicine, Bronx, NY, USA \\ ${ }^{\mathrm{c}}$ Department of Veterinary Clinical Sciences, Purdue University, West Lafayette, IN, USA \\ ${ }^{\mathrm{d}}$ Department of Epidemiology \& Population Health, Albert Einstein College of Medicine, Bronx, NY, USA \\ e Purdue University Center for Cancer Research, West Lafayette, IN, USA
}

Received 13 November 2018

Accepted 12 January 2019

\begin{abstract}
.
Background: B7x (B7-H4/B7S1/VTCN1), an inhibitory immune checkpoint molecule is a potential therapeutic target owing to its immunosuppressive effect and well-known expression in cancers. Immune checkpoints in canine bladder cancer are largely undefined. Here, we report the first evaluation on expression of B7x in spontaneous canine invasive bladder cancer, a novel model system for the study of invasive human urothelial carcinoma.

Objective: This work aims to study expression of immune checkpoint B7x in spontaneous canine invasive bladder cancer. Methods: RNA-seq analysis was performed to determine B7x expression in tumor versus normal bladder. Gene ontology (GO) study was used to explore the biological role of B7x. B7x protein expression was evaluated by immunohistochemistry (IHC). TCGA and GTEx were used to examine B7x expression in 599 human bladder urothelial carcinoma (BLCA).

Results: RNA-seq analysis indicated 5.72 and 7.04 fold up regulation of B7x in tumors, using DESeq2 and edge R respectively $(p<0.00008)$. B7x was closely associated with immune processes in $\mathrm{GO}$ analysis. IHC results revealed $60 \%$ of cases as B7x positive. B7x intensity was scored as negative in $40 \%(n=20 / 50)$, low in $24 \%(n=12 / 50)$, medium in $14 \%(n=7 / 50)$ and high in $22 \%(n=11 / 50)$ of cases. In human BLCA, B7x expression was significantly associated with worse overall survival $(p=0.02)$.

Conclusions: Our results suggest that B7x is over expressed in canine bladder cancer. Thus canine model can be vital in advancing the translational research on $\mathrm{B} 7 \mathrm{x}$, a new potential therapeutic target in human bladder cancer.
\end{abstract}

Keywords: Canine bladder cancer, immune checkpoint, immunotherapy, human bladder urothelial carcinoma

\footnotetext{
${ }^{*}$ Correspondence to: Xingxing Zang, Department of Microbiology and Immunology, Department of Urology, Albert Einstein
} 


\section{ABBREVIATIONS}

\author{
$\mathrm{iUC}=$ Invasive urothelial carcinoma \\ $\mathrm{IHC}=$ immunohistochemistry \\ $\mathrm{BCG}=$ Bacillus Calmette-Guerin \\ $\mathrm{GO}=$ gene ontology \\ TCGA = The Cancer Genome Atlas \\ GTEx $=$ Genotype Tissue Expression project \\ $\mathrm{BLCA}=$ bladder urothelial carcinoma
}

\section{INTRODUCTION}

A better understanding of tumor immune biology has revolutionized the cancer therapy field in recent years. This paradigm shift from tumor itself to the host's immune system has led to remarkable progress in the field of immunotherapy [1]. One of the most notable therapeutic manipulations of the immune system involves the immune checkpoint molecules. The field has witnessed great success with the use of checkpoint inhibitors, several of which are FDA approved for treatment in cancer.

B7-CD28, the most well studied family of immune checkpoints, plays an important role in $\mathrm{T}$ cell co-stimulation and co-inhibition [2]. Phylogenetically, this growing ligand-receptor family of B7-CD28 can be divided into three groups; Group I that includes B7-1/B7-2/CD28/CTLA4 and $\mathrm{B} 7 \mathrm{~h} / \mathrm{ICOS}$, Group II consisting PD-L1/PDL2/PD-1 and Group III comprising B7-H3 (CD276), B7x (B7-H4/B7S1/VTCN1), and HHLA2/TMIGD2 (IGPR-1/CD28H) [3].

B7x was first discovered in 2003 and is a relatively new member of the B7 family [4-6]. It plays a role in T-cell co-inhibition and shares $15-25 \%$ homology with other B7 members [3]. Its expression in normal tissues is limited, however it is detected in a myriad of cancers including human bladder cancer $[7,8]$. B7X is associated with increased TNM stage, pathological grade and poorer outcomes with urothelial carcinoma [9]. Additionally, serum B7x levels are high in patients with urothelial carcinoma [10]. Its immunosuppressive effect and widespread expression in cancers makes $\mathrm{B} 7 \mathrm{x}$ an important target for immunotherapy.

As immunotherapies are emerging, relevant preclinical models in which the cancer and the level of host immunocompetence are similar between the model and the human condition are essential. Reports of the expression of immune checkpoints like PDL1 in canine tumors has led to a surge in interest in developing canine-specific antibodies for checkpoint blockade in translational research $[11,12]$. It is particularly advantageous to study specific forms of naturally-occurring canine cancer because of the many similarities shared with human cancer including incidence, environmental risk factors, cancer behavior, and response to therapy [13]. Dogs have been emphasized as a clinically relevant model to aid the development of personalized medicine strategies for cancer [14]. Naturally-occurring canine bladder cancer (invasive urothelial carcinoma, iUC) closely resembles human muscle invasive bladder cancer and is considered a relevant model to complement traditional models for bladder cancer research [15]. Canine and human invasive bladder cancer share many features including physiological age of onset, clinical symptoms, histopathology, heterogeneity, metastatic behavior, molecular subtypes, epigenetic features and treatment response $[15,16]$. Moreover, similarities between enrichment patterns of gene expression have also been observed in canine and human bladder cancer including genes involved in the p53 pathway [17].

Immune checkpoints in canine bladder cancer are largely unknown. For the first time, we examined the immune checkpoint molecule $\mathrm{B} 7 \mathrm{x}$ in canine bladder cancer. In this study, using immunohistochemistry and RNA-seq we report over expression of B7x in tissues from canine bladder cancer. Our findings suggest that the canine bladder cancer model can be vital in furthering the studies on B7x. This may ultimately help in leading this potential therapeutic target to become a therapy option in human cancer.

\section{MATERIALS AND METHODS}

\section{Canine invasive bladder cancer samples}

With the approval of Purdue Animal Care and Use Committee, canine tissues were collected for the work. RNA-seq data from a previously published study [18] were mined to determine B7x expression. The RNA-seq work was performed on 29 cystoscopic biopsy samples of iUC from treatment-naive dogs, and on 4 normal bladder mucosa samples collected from dogs being euthanized due to non-bladder related conditions. A set of 50 formalin fixed paraffin embedded iUC samples (from 50 distinct dogs), and four normal bladder tissues were assembled for immunohistochemistry (IHC). Clinical and pathological information for the cases was compiled from the medical records in the Purdue University Veterinary 
Teaching Hospital. Data collected included age, gender, TNM staging at diagnosis and at death, survival days, and outcome with the first treatment.

\section{RNA-seq analyses}

The RNA-seq data from previous canine iUC work [18] were processed, and statistical analyses were conducted using Strand NGS, v3.1, Build 235027, (C) Strand Life Sciences, Bangalore, India) [19]. Raw RNA-Seq data were subjected to normalization (separately using TMM and DESeq), filtered by read metrics, and subjected to quantification. Pairwise comparison was performed using edge R (on TMM normalized data) and DESeq2 (on DESeq normalized data) with Benjamini-Hochberg FDR multiple testing correction ( $\mathrm{p}$ corr $<0.05$; 2-fold or higher change) comparing normal mucosa/urothelial layer samples versus canine invasive bladder cancer samples. Differentially expressed genes identified using both methodologies were pooled for subsequent analyses. BoxWhisker plot was used to visualize the difference in enrichment of $\mathrm{B} 7 \mathrm{x}$ in normal and tumor samples.

\section{Cell culture for immunohistochemistry controls}

Human breast cancer cell line SKBR3 cultured in RPMI was used as a $\mathrm{B} 7 \mathrm{x}$ positive control for immunohistochemistry. Untransfected 3T3 cells grown in complete DMEM media were used as negative control. Cells were fixed in $10 \%$ neutral buffered formalin overnight and encapsulated in HistoGel (Thermo-Scientific). These samples were paraffin embedded and cut onto slides for staining.

\section{IHC}

Prior to staining, slides were baked at $60^{\circ} \mathrm{C}$ overnight. FFPE sections were de-paraffinized and hydrated by washing in xylene and decreasing concentrations of ethanol $(100 \%, 95 \%)$. Antigen retrieval was performed by first boiling the samples in signal stain citrate unmasking solution (Cell signaling technology) for 2 mins. Then, the slides were placed in a steamer for additional 20 minutes. Slides were incubated in $3 \%$ hydrogen peroxide for 10 mins to block endogenous peroxidase. Non-specific binding of IgG was blocked using serum-free Protein block (Dako). Sections were labeled with primary antibody anti- human B7x (clone D1M8I, Cell signaling technology) overnight at $4^{\circ} \mathrm{C}$. Secondary HRP conjugated goat anti- rabbit (Cell signaling technology) was added subsequently, followed by addition of chromogen DAB (Cell signaling technology) and hematoxylin nuclear counterstaining. Images were acquired using Zeiss Axio Observer CLEM microscope. The intensity of the immunoreactivity was scored as 0 (none), 1 (low), 2 (medium), or 3 (high).

\section{Biological functions modulated by B7x in canine urothelial carcinoma}

Correlation analyses were performed on the differentially expressed genes identified. The genes that positively correlated with the expression of B7x (i.e. increased expression of the gene of interest and B7x) were compiled and only the genes that strongly correlated with B7x was selected (correlation coefficient $>0.6, n=457)$. GO analyses was conducted on these genes. The genes identified in GO terms associated with immune functions and signaling were hierarchically clustered with respect to expression of B7x $(n=97)$.

\section{B7x in human bladder urothelial carcinoma}

To further assess the importance of B7x expression in human bladder cancer, $\mathrm{B} 7 \mathrm{x}$ mRNA expression in human bladder urothelial carcinoma (BLCA) in TCGA and GTEx were analyzed by Gene Expression Profiling Interactive Analysis. The association between B7x expression and overall survival was analyzed.

\section{Statistical analyses}

$\chi 2$ or Fisher's exact test was appropriately used to compare the frequency distribution of categorical variables, including staging and treatment outcome, between no or low B7x expression ("low expression" group) and medium or high B7x intensity ("high expression" group). Wilcoxon rank-sum test was used to test the difference in continuous variables, including the age at diagnosis, between B7x groups. For survival analysis, The Kaplan-Meier survival curves of B7x "low expression" group and B7x "high expression" group were compared using the log-rank test. All statistical tests were two-sided. $P$ values $\leq 0.05$ were considered significant. The analysis was done using the SAS 9.4 software (SAS Institute Inc.). Multivariate Cox model analysis was also conducted to estimate the hazard ratio of death for the high versus 
low B7x expression groups, with additional adjustment of age, gender, stage and treatment.

\section{RESULTS}

\section{B7x mRNA is highly upregulated in canine invasive bladder cancer}

RNA was processed and analyzed using Strand NGS as described previously [18, 19]. B7x was found to be 5.72 fold upregulated using DESeq2 $(p=0.000000017572415)$ and 7.04 upregulated using edge $\mathrm{R}$ ( $p=0.00007840038)$. The BoxWhisker plot demonstrates the enrichment of $\mathrm{B} 7 \mathrm{x}$ in canine invasive bladder cancer samples as compared to normal urothelial samples (Fig. 1a).

\section{Expression of B7x protein in canine invasive bladder cancer}

Tumors from fifty canine bladder cancer cases were assessed for $\mathrm{B} 7 \mathrm{x}$ protein expression using immunohistochemistry (Fig. 1b). Of the total 50 cases, 30 cases were considered positive for $\mathrm{B} 7 \mathrm{x}$ expression with varying levels of IHC intensity. The intensity was scored as 0 in $40 \%(n=20 / 50), 1$ in $24 \%(n=12 / 50), 2$ in $14 \%(n=7 / 50)$ and 3 in $22 \%$ $(n=11 / 50)$. Overall, $60 \%$ of total cases showed B7x protein expression and were considered as $\mathrm{B} 7 \mathrm{x}$ positive. The B7x expression in 4 normal bladder sections was scored as 0 in 3/4 and as 2 in 1/4, therefore, $25 \%$ of normal bladders were considered $\mathrm{B} 7 \mathrm{x}$ positive.

\section{B7x expression and clinical outcome}

The clinical features of cases analyzed with IHC are summarized in Table 1. For comparison, the dogs with no or low B7x expression ("low expression" group) group were compared to those with medium or high B7x intensity ("high expression" group). There was no correlation between B7x expression (low versus high group) and the clinical features (Table 1).

B7x expression was similar across disease stages. At diagnosis, $13 \%$ of dogs with low B7x expression had metastases ( 1 case with NOM1, 3 cases with N1M1 stage disease). In the high B7x group, 17\% of dogs had metastases at diagnosis ( 1 case with N1M0, 2 cases with N1M1 stage disease) $(p=0.72)$. At death, $33 \%$ and $23 \%$ of the cases with high and low B7x expression, respectively, still had NOM0 disease.

The owners of 46 dogs elected to have their dog treated for its bladder cancer. Standard of care treatment in dogs typically includes cytotoxic chemotherapy (e.g. vinblastine, mitoxantrone, carboplatin), which is often given concurrently with a nonsteroidal anti-inflammatory drug (NSAID) as NSAIDs enhance the activity of chemotherapy in canine bladder cancer $[15,16]$. Some pet owners opt for a more conservative treatment consisting of a NSAID alone. The type of treatment, outcome of treatment, and overall survival were similar between the low and high B7x expression groups (Table 1). Second line therapy was administered to $79 \%$ of dogs in the low expression group and to $71 \%$ of dogs in the high expression group. In exploratory analyses in the treated dogs $(n=46)$, the high expression group $(n=17)$ had a median overall survival of 298 days. The dogs in the low expression group $(n=29)$ had a median survival of 270 days $(p=0.23)$. Furthermore, Multivariate Cox model analysis was conducted and after additional adjustment of age, gender, stage and treatment, the hazard ratio of death for high B7x expression group compared with low expression group was determined as $0.647(0.332$ to 1.263) $(p=0.20)$.

\section{B7x closely related to immune function genes in canine $i U C$}

To elucidate the biological role of $\mathrm{B} 7 \mathrm{x}$ in canine iUC, gene ontology (GO) analysis was performed. Pearson's correlation analyses were conducted on the differentially expressed genes. This was used to create a list of genes that strongly and positively correlated with B7x expression $(n=457)$ (correlation coefficient $>0.6$ ). GO analyses was conducted on these genes and the genes identified in GO terms associated with immune functions and signaling are indicated in the bar diagram (Fig. 2a). These were hierarchically clustered with respect to expression of B7x $(n=97)$. As illustrated in the Fig. 2b, most of the genes positively correlated with $\mathrm{B} 7 \mathrm{x}$.

\section{B7x mRNA expression was associated with poor overall survival in human bladder cancer}

We finally examined B7x mRNA expression and clinical significance in human BLCA. TCGA and GTEx contained a total of 599 BLCA samples. These samples were separated into two groups according to their B7x expression levels: the B7x high group (top 25\%, $\mathrm{N}=298$ ) and the $\mathrm{B} 7 \mathrm{x}$ low group (the rest $75 \%, \mathrm{~N}=301$ ). The result revealed that the $\mathrm{B} 7 \mathrm{x}$ high 


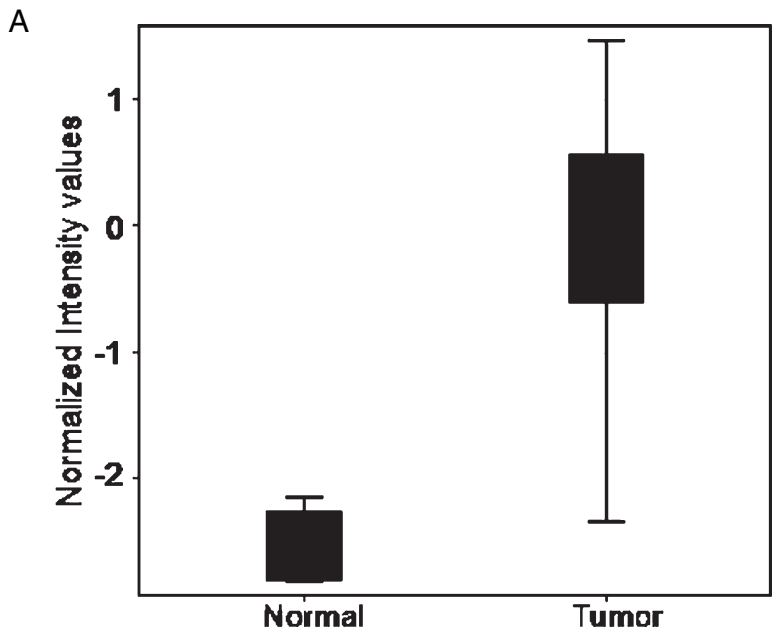

B

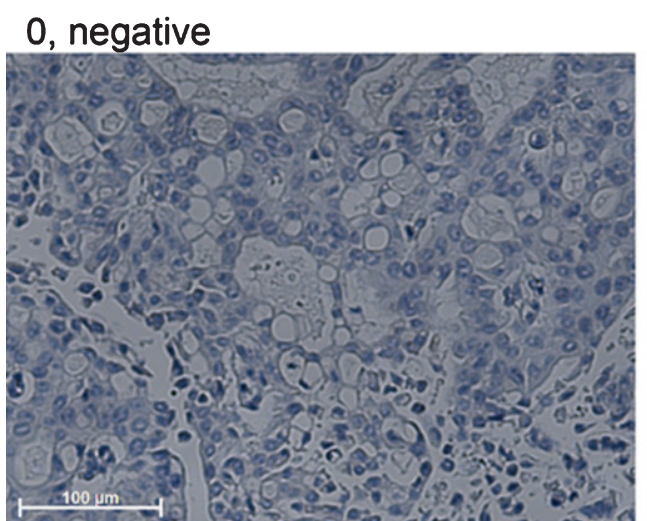

\section{1 low}
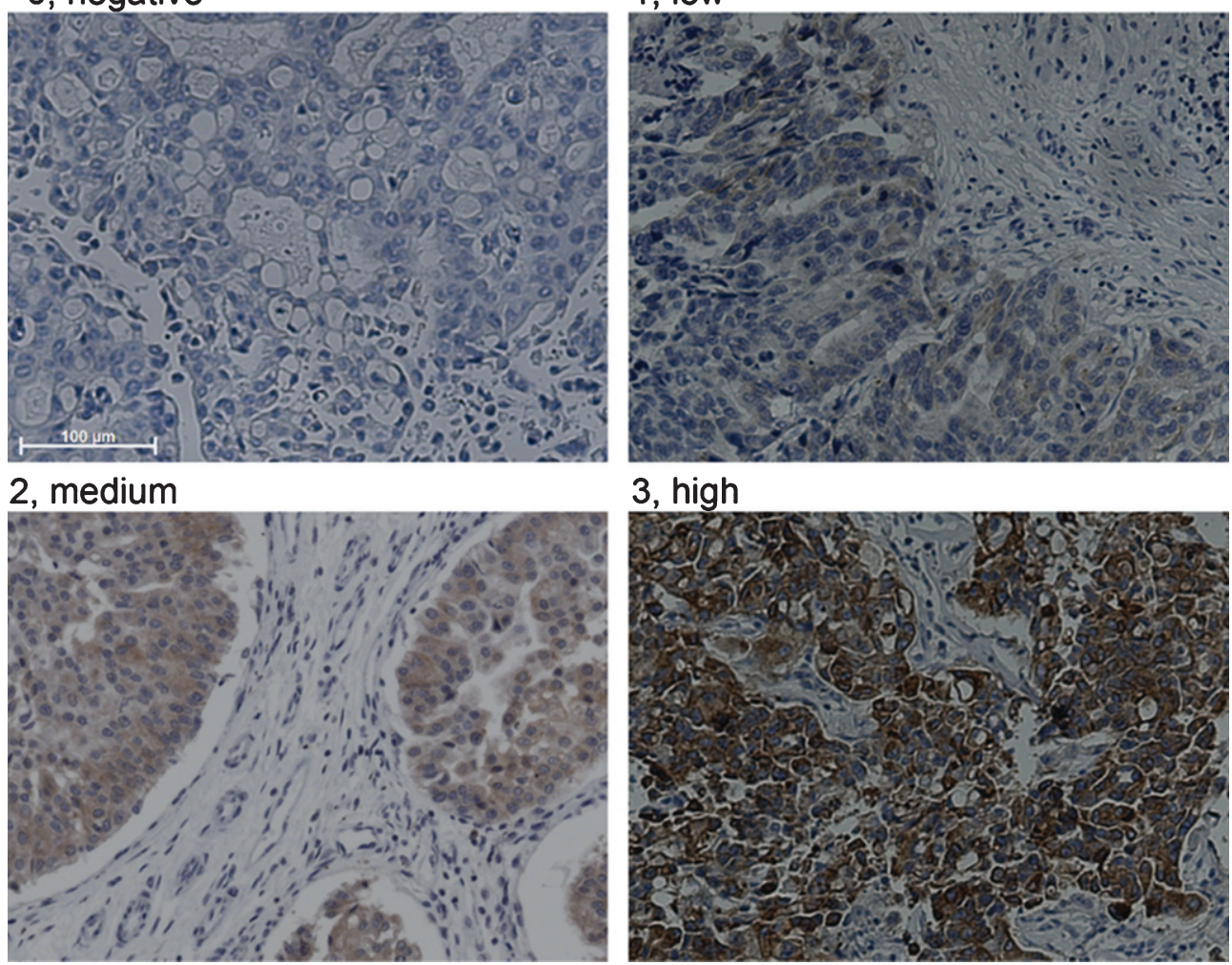

\section{3 , high}

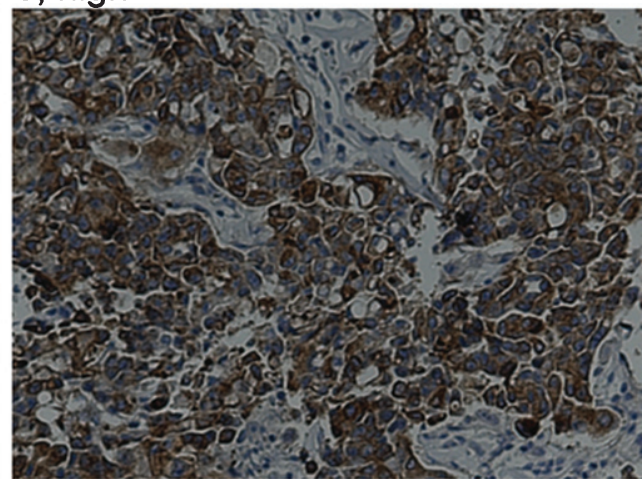

Fig. 1. B7x expression in canine bladder cancer. a. RNA-seq data was processed and analyzed using Strand NGS. BoxWhisker plot was used to show upregulation of B7x in canine iUC versus normal bladder. b. The intensity of B7x immunostaining was graded as follows: 0 ,

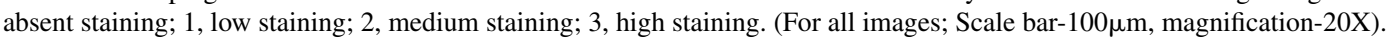

group had significant poorer overall survival than the B7x low group $(p=0.02)$ (Fig. 3), demonstrating B7x mRNA expression was associated with poor clinical outcome in human BLCA.

\section{DISCUSSION}

Immunotherapy has been a long-standing treatment approach for patients with bladder cancer. For 
Table 1

Comparison of clinical characteristics between B7x low and B7x high groups

\begin{tabular}{lccc}
\hline & \multicolumn{2}{c}{ Frequency no. $(\%)$} & \multirow{2}{*}{$p$-value } \\
\cline { 2 - 3 } Ag7x Low $(\mathrm{N}=32)$ & B7x high (N=18) & \\
Sex & $11.5(9.2-12.85)$ & $10.05(9.1-11.2)$ & 0.18 \\
$\quad$ Male & $11(34.38)$ & $7(38.89)$ & 0.75 \\
Female & $21(65.63)$ & $11(61.11)$ & \\
Tumor stage* & & & \\
T1 & $1(3.23)$ & $1(5.56)$ & 0.83 \\
T2 & $26(83.87)$ & $16(88.89)$ & \\
T3 & $4(12.90)$ & $1(5.56)$ & \\
Lymph node infiltration & $28(90.32)$ & $15(83.33)$ & 0.38 \\
N0 & $3(9.68)$ & $3(16.67)$ & \\
N1 & & & \\
Disease stage & $27(87.10)$ & $16(88.89)$ & 1.00 \\
M0 (no distant metastases) & $4(12.90)$ & $2(11.11)$ & \\
M1 (distant metastases) & & & \\
Primary treatment & $21(66)$ & $11(61)$ & 0.88 \\
Chemotherapy \pm NSAID & $6(19)$ & $5(28)$ & \\
Single agent NSAID & $2(6)$ & $1(6)$ & \\
Experimental drug & $3(9)$ & $1(6)$ & \\
No treatment & $6(20.69)$ & $3(18.75)$ & 0.71 \\
Primary treatment outcome & $17(58.62)$ & $8(50.00)$ & \\
PD (progressive disease) & $6(20.69)$ & $5(31.25)$ & \multirow{2}{*}{$0.23^{* * *}$} \\
SD (stable disease) & 270 & 298 & \\
PR (partial response) & & & \\
Median survival of treated dogs (days) & & & \\
\hline
\end{tabular}

*TNM stage was assigned following World Health Organization criteria for canine tumors. (TNM Classification of Tumours in Domestic Animals, Geneva, Switzerland: World Health Organization, 1980). Please note that T2 tumors in the canine staging system are equivalent to $\mathrm{T} 3$ tumors in humans. i.e. are invasive. In one dog in the low risk group, the TNM stage was not defined. $* * p$-value for the log-rank test.

more than 40 years since its first introduction in 1976, intravesical treatment with BCG has been a standard of care for non-invasive bladder cancer [20]. Although, BCG has been one of the most successful immunotherapies, about $40 \%$ patients post therapy will progress to muscle invasive bladder cancer [21]. The introduction of checkpoint immunotherapy marked a major milestone for bladder cancer. In recent years, anti- PD-1/PD-L1 treatment has gained FDA approval, making it the first immunotherapy since the BCG therapy to be approved in bladder cancer [22]. Numerous clinical trials are underway to evaluate the effect of checkpoint inhibitors alone and in combination, across multiple disease states from non-invasive to metastatic bladder cancer [23, 24].

Identifying novel targets in immunotherapy is an unmet need, because despite clinical success, many patients do not respond to treatment, or some patients have a good initial response followed by acquired resistance to the treatment [25]. There is a growing interest in identifying resistance mechanisms and discovering new targets for combination therapy. This entails the development of or the identification of better and more reliable animal models that recapitulate human conditions. Better models offer a greater chance to accurately predict the safety and efficacy of new treatments in humans, to thereby improve the success rates in human trials, and to potentially shorten the time from pre-clinical to clinical trials. This is crucial for reducing the global cancer burden as well as substantially reducing costs incurred in the clinical trials.

Several studies have revealed the potential of $\mathrm{B} 7 \mathrm{x}$ as a crucial immunotherapeutic target. B7x blockade has yielded promising results in mouse colon and breast cancer models [26]. In a humanized mouse model of ovarian cancer, tumor growth was delayed when animals were treated with anti-B7x single chain fragments variable (scFv) [27]. Similarly, anti-B7x CAR T-cell therapy significantly reduced tumor burden in a human ovarian tumor xenograft model [28]. Combining gemcitabine with B7x deficiency in B7x knockout host also showed better response than when treated with gemcitabine alone [29]. Additionally, in murine pulmonary metastasis model, inducing expression of $\mathrm{B} 7 \mathrm{x}$ resulted in increased tumor 
A

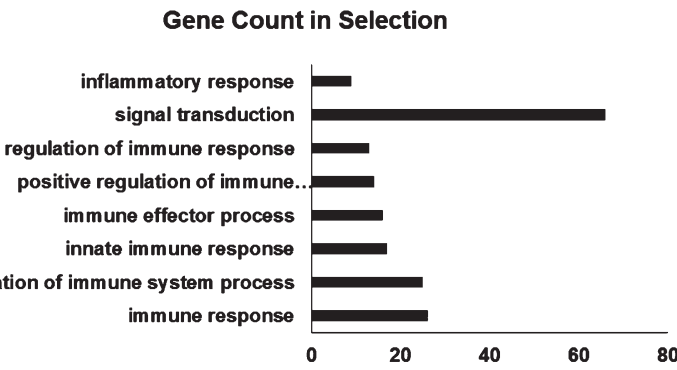

$\mathrm{B}$

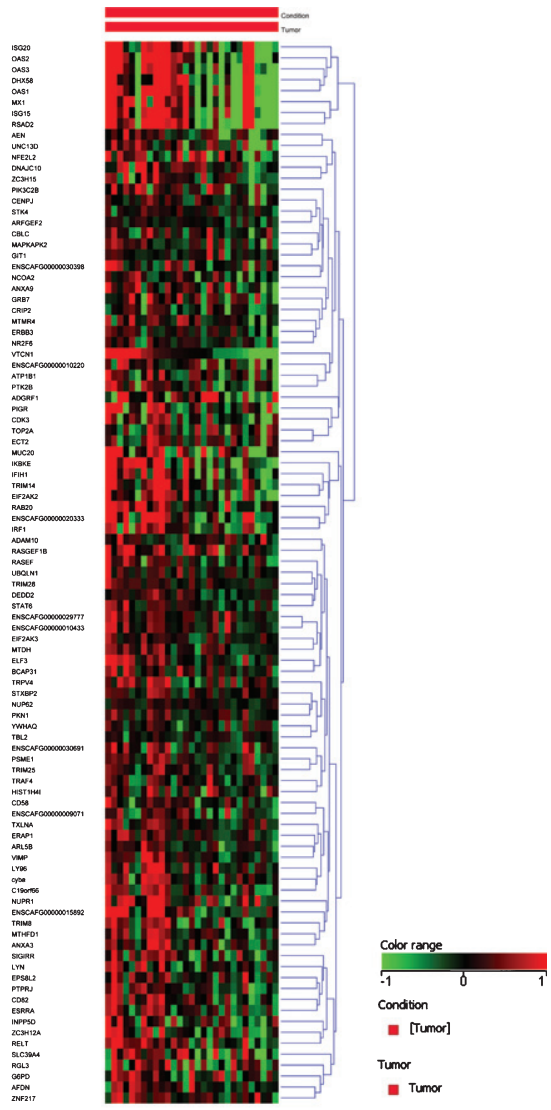

Fig. 2. B7x was closely related to immune functions in canine iUC. a. The bar diagram indicated the genes identified in Gene ontology (GO) analysis and their functional role. Most genes are associated with immune functions and signaling. b. Heat map depicted that most genes positively correlated with B7x expression. Each column represents a tumor sample, and each row represents a gene.

progression and led to immune suppression in the tumor microenvironment [30].

We report over expression of the immune checkpoint $\mathrm{B} 7 \mathrm{x}$ in canine bladder cancer. From our RNA-seq analysis, we observed a 5.72 and 7.04 fold upregulation of B7x using DESeq2 and edge $R$ respectively. Using traditional immunohistochemistry, we showed $\mathrm{B} 7 \mathrm{x}$ protein expression in sixty percent of cases in this study. Using TCGA and GTEx, we examined B7x expression in 599 human bladder urothelial carcinoma (BLCA) and showed that B7x expression was significantly associated with worse overall survival in human BLCA. Further building on published findings signifying $\mathrm{B} 7 \mathrm{x}$ as a potential therapeutic target.

Although the degree of $\mathrm{B} 7 \mathrm{x}$ expression did not correlate with pathologic or clinical outcomes in this group of dogs, patterns may emerge when larger sample sets are examined. It is recognized that additional tumor-immune pathways could be involved and could contribute to the clinical outcome. Therefore examining multiple immune checkpoints and regulatory molecules will be pursued in future studies. In addition, the association between the outcome of a specific treatment protocol and B7x expression could be of importance. In the current study, dogs were treated with medical therapies because cystectomy is not typically performed in dogs. Although the therapies were similar across the cohort, the specific treatment protocol did vary from case to case.

With emergence of new targets for immunotherapy, there is a greater demand for relevant pre-clinical models to assess the efficacy and safety of these therapies. Our study underscores the importance of the naturally-occurring canine model of invasive bladder cancer as a complementary model to experimental systems, and how this canine model could prove crucial for expediting the studies on new immunotherapy targets such as B7x. This is vital for advancing translational research and could help lead targeted 


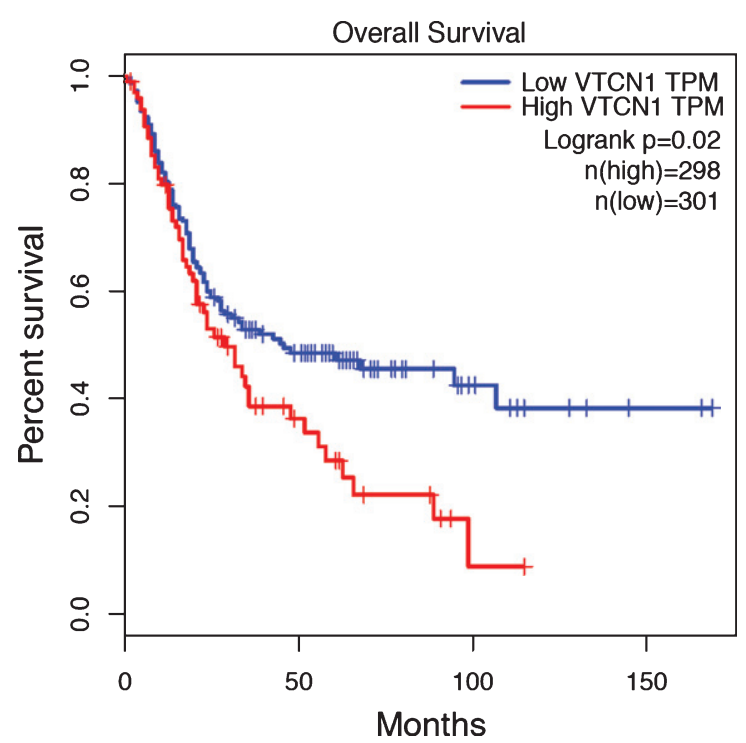

Fig. 3. B7x mRNA expression was associated with poor overall survival in human bladder urothelial carcinoma. B7x mRNA in TCGA and GTEx datasets of human bladder urothelial carcinoma was analyzed using Gene Expression Profiling Interactive Analysis. The B7x high group (top $25 \%, \mathrm{~N}=298$ ) indicated in red showed a significant worse overall survival than the B7x low group (the rest $75 \%, \mathrm{~N}=301$ ) in blue, $p=0.02$.

therapies in clinical trials in humans, thereby benefiting both humans as well as dogs alike.

\section{FUNDING}

D. Chand is supported by the Thanks to Scandinavia/Borge endowment. This work was supported by NIH R01CA175495 and R01DK100525 (X. Zang).

\section{CONFLICT OF INTEREST}

$\mathrm{X}$. Zang is an inventor on patent number US 9447186 B2, which covers the topic of cancer immunotherapy targeting $\mathrm{B} 7 \mathrm{x}$. The remaining authors declare no competing financial interests.

\section{REFERENCES}

[1] Miller JF, Sadelain M. The journey from discoveries in fundamental immunology to cancer immunotherapy. Cancer Cell. 2015;27(4):439-49.

[2] Zang X, Allison JP. The B7 family and cancer therapy: Costimulation and coinhibition. Clin Cancer Res. 2007;13(18 Pt 1):5271-9.

[3] Janakiram M, Shah UA, Liu W, Zhao A, Schoenberg MP, Zang X. The third group of the B7-CD28 immune checkpoint family: HHLA2, TMIGD2, B7x, and B7-H3. Immunol Rev. 2017;276(1):26-39.
[4] Zang X, Loke P, Kim J, Murphy K, Waitz R, Allison JP. B7x: A widely expressed B7 family member that inhibits $\mathrm{T}$ cell activation. Proc Natl Acad Sci U S A. 2003;100(18):1038892.

[5] Prasad DV, Richards S, Mai XM, Dong C. B7S1, a novel B7 family member that negatively regulates $\mathrm{T}$ cell activation. Immunity. 2003;18(6):863-73.

[6] Sica GL, Choi IH, Zhu G, Tamada K, Wang SD, Tamura $\mathrm{H}$, et al. B7-H4, a molecule of the B7 family, negatively regulates T cell immunity. Immunity. 2003;18(6): 849-61.

[7] Sankin A, Narasimhulu D, John P, Gartrell B, Schoenberg $\mathrm{M}$, Zang X. The expanding repertoire of targets for immune checkpoint inhibition in bladder cancer: What lies beneath the tip of the iceberg, PD-L1. Urol Oncol. 2017.

[8] Zang X, Thompson RH, Al-Ahmadie HA, Serio AM, Reuter VE, Eastham JA, et al. B7-H3 and B7x are highly expressed in human prostate cancer and associated with disease spread and poor outcome. Proc Natl Acad Sci U S A. 2007;104(49):19458-63.

[9] Fan M, Zhuang Q, Chen Y, Ding T, Yao H, Chen L, et al. B7-H4 expression is correlated with tumor progression and clinical outcome in urothelial cell carcinoma. Int J Clin Exp Pathol. 2014;7(10):6768-75.

[10] Liu WH, Chen YY, Zhu SX, Li YN, Xu YP, Wu XJ, et al. B7$\mathrm{H} 4$ expression in bladder urothelial carcinoma and immune escape mechanisms. Oncol Lett. 2014;8(6):2527-34.

[11] Maekawa N, Konnai S, Ikebuchi R, Okagawa T, Adachi M, Takagi S, et al. Expression of PD-L1 on canine tumor cells and enhancement of IFN-gamma production from tumor-infiltrating cells by PD-L1 blockade. PLoS One. 2014;9(6):e98415.

[12] Coy J, Caldwell A, Chow L, Guth A, Dow S. PD-1 expression by canine $\mathrm{T}$ cells and functional effects of PD-1 blockade. Vet Comp Oncol. 2017;15(4):1487-502.

[13] Paoloni M, Khanna C. Translation of new cancer treatments from pet dogs to humans. Nat Rev Cancer. 2008;8(2):14756.

[14] Khanna C, Lindblad-Toh K, Vail D, London C, Bergman P, Barber $\mathrm{L}$, et al. The dog as a cancer model. Nat Biotechnol. 2006;24(9):1065-6.

[15] Fulkerson CM, Dhawan D, Ratliff TL, Hahn NM, Knapp DW. Naturally occurring canine invasive urinary bladder cancer: A complementary animal model to improve the success rate in human clinical trials of new cancer drugs. Int J Genomics. 2017;2017:6589529.

[16] Knapp DW, Ramos-Vara JA, Moore GE, Dhawan D, Bonney PL, Young KE. Urinary bladder cancer in dogs, a naturally occurring model for cancer biology and drug development. ILAR J. 2014;55(1):100-18.

[17] Dhawan D, Paoloni M, Shukradas S, Choudhury DR, Craig BA, Ramos-Vara JA, et al. Comparative gene expression analyses identify luminal and basal subtypes of canine invasive urothelial carcinoma that mimic patterns in human invasive bladder cancer. PLoS One. 2015;10(9): e0136688.

[18] Dhawan D, Hahn NM, Ramos-Vara JA, Knapp DW. Naturally-occurring canine invasive urothelial carcinoma harbors luminal and basal transcriptional subtypes found in human muscle invasive bladder cancer. PLoS Genet. 2018;14(8):e1007571.

[19] Brunskill EW, Potter SS. RNA-Seq defines novel genes, RNA processing patterns and enhancer maps for the early stages of nephrogenesis: Hox supergenes. Dev Biol. 2012;368(1):4-17. 
[20] Morales A, Eidinger D, Bruce AW. Intracavitary Bacillus Calmette-Guerin in the treatment of superficial bladder tumors. J Urol. 1976;116(2):180-3.

[21] Boegemann M, Aydin AM, Bagrodia A, Krabbe LM. Prospects and progress of immunotherapy for bladder cancer. Expert Opin Biol Ther. 2017;17(11):1417-31.

[22] Zhou TC, Sankin AI, Porcelli SA, Perlin DS, Schoenberg MP, Zang X. A review of the PD-1/PD-L1 checkpoint in bladder cancer: From mediator of immune escape to target for treatment. Urol Oncol. 2017;35(1):14-20.

[23] A Phase1/2,Open-label study of nivolumab monotherapy or nivolumab combined with ipilimumab in subjects with advanced or metastatic solid tumors. ClinicalTrials.gov Identifier: NCT01928394.

[24] A Phase1 Study of (Cabozantinib) Plus Nivolumab (CaboNivo) alone or in combination with ipilimumab (CaboNivoIpi) in patients with advanced/metastatic urothelial carcinoma and other genitourinary tumors. ClinicalTrials.gov Identifier: NCT02496208.

[25] Sharma P, Hu-Lieskovan S, Wargo JA, Ribas A. Primary, Adaptive, and Acquired Resistance to Cancer Immunotherapy. Cell. 2017;168(4):707-23.
[26] Jeon H, Vigdorovich V, Garrett-Thomson SC, Janakiram M, Ramagopal UA, Abadi YM, et al. Structure and cancer immunotherapy of the B7 family member B7x. Cell Rep. 2014;9(3):1089-98.

[27] Dangaj D, Lanitis E, Zhao A, Joshi S, Cheng Y, Sandaltzopoulos R, et al. Novel recombinant human b7-h4 antibodies overcome tumoral immune escape to potentiate T-cell antitumor responses. Cancer Res. 2013;73(15):48209.

[28] Smith JB, Lanitis E, Dangaj D, Buza E, Poussin M, Stashwick C, et al. Tumor regression and delayed onset toxicity following B7-H4 CAR T cell therapy. Mol Ther. 2016;24(11):1987-99.

[29] Leung J, St-Onge P, Stagg J, Suh WK. Synergistic effects of host B7-H4 deficiency and gemcitabine treatment on tumor regression and anti-tumor $\mathrm{T}$ cell immunity in a mouse model. Cancer Immunol Immunother. 2017;66(4):491-502.

[30] Ohaegbulam KC, Liu W, Jeon H, Almo SC, Zang X. Tumor-expressed immune checkpoint $\mathrm{B} 7 \mathrm{x}$ promotes cancer progression and antigen-specific CD8 $\mathrm{T}$ cell exhaustion and suppressive innate immune cells. Oncotarget. 2017;8(47):82740-53. 